Pacific

Journal of

Mathematics

RECONSTRUCTION FROM KOSZUL HOMOLOGY AND APPLICATIONS TO MODULE AND DERIVED CATEGORIES

\author{
RYO TAKAHASHI
}




\title{
RECONSTRUCTION FROM KOSZUL HOMOLOGY AND APPLICATIONS TO MODULE AND DERIVED CATEGORIES
}

\author{
RYO TAKAHASHI
}

\begin{abstract}
Let $R$ be a commutative noetherian ring and $M$ a finitely generated $R$-module. In this paper, we reconstruct $M$ from its Koszul homology with respect to a suitable sequence of elements of $\boldsymbol{R}$ by taking direct summands, syzygies and extensions, and count the number of those operations. Using this result, we consider generation and classification of certain subcategories of the category of finitely generated $\boldsymbol{R}$-modules, its bounded derived category and the singularity category of $R$.
\end{abstract}

\section{Introduction}

For the past five decades, a lot of classification theorems of subcategories of abelian categories and triangulated categories have been given in ring theory, representation theory, algebraic geometry and algebraic topology; see, for instance, [Balmer 2002; 2005; Benson et al. 2011; Dao and Takahashi 2014; Friedlander and Pevtsova 2007; Gabriel 1962; Hopkins and Smith 1998; Hovey 2001; Krause 2008; Krause and Stevenson 2013; Neeman 1992; Stevenson 2014; Takahashi 2010; 2013; Thomason 1997]. Reconstruction of an object from its support in the spectrum of a suitable commutative ring plays a crucial role in the proofs of those theorems.

The notion of dimension for triangulated categories was introduced by Bondal and Van den Bergh [2003] and by Rouquier [2008]; analogues for abelian categories were introduced by Dao and Takahashi [2011;2012a]. They essentially indicate the number of extensions necessary to build all objects out of a single object. There are many related studies; for example, see [Aihara and Takahashi 2011; Avramov et al. 2010a; Ballard et al. 2012; Bergh et al. 2010; Burke et al. 2012; Christensen 1998; Krause and Kussin 2006; Dao and Takahashi 2012b; Oppermann 2009; Orlov 2009b; Rouquier 2006; Schoutens 2003; Takahashi 2009].

Takahashi was partially supported by JSPS Grant-in-Aid for Young Scientists (B) 22740008 and by JSPS Postdoctoral Fellowships for Research Abroad.

MSC2010: 18E30, 18E35, 13C60, 13D09.

Keywords: Koszul complex, Koszul homology, module category, derived category, singularity category, resolving subcategory, thick subcategory. 
In this paper, we study reconstructing a given module from its Koszul homology and counting the number of necessary operations. Our main result is the following theorem.

Theorem 1.1. Let $R$ be a commutative noetherian ring, and let $M$ be a finitely generated $R$-module. Let $\boldsymbol{x}=x_{1}, \ldots, x_{n}$ be a sequence of elements of $R$ such that $M$ is locally free on $\mathrm{D}(\boldsymbol{x})$. Then there exists a positive integer $k$ such that the Koszul complex $\mathrm{K}\left(\boldsymbol{x}^{k}, M\right)$ is equivalent to a complex of finitely generated $R$-modules

$$
\left(0 \rightarrow N \rightarrow P_{n-1} \rightarrow \cdots \rightarrow P_{0} \rightarrow 0\right)
$$

where $P_{0}, \ldots, P_{n-1}$ are projective and $M$ is a direct summand of $N$. In particular, $M$ can be built out of the Koszul homologies $\mathrm{H}_{0}\left(\boldsymbol{x}^{k}, M\right), \ldots, \mathrm{H}_{n}\left(\boldsymbol{x}^{k}, M\right)$ by taking $n$ syzygies, $n$ extensions and 1 direct summand.

Note that since the free locus of a finitely generated $R$-module is an open subset of Spec $R$ in the Zariski topology, there exist many such sequences $\boldsymbol{x}$ that satisfy the assumption of the theorem. We shall prove a more general result in Theorem 3.1.

Theorem 1.1 has a lot of applications. To state some of them, we fix notation. Let $\bmod R$ be the category of finitely generated $R$-modules and $\mathbf{D}_{\mathrm{b}}(R)$ the bounded derived category of $\bmod R$. We denote by $\mathbf{D}_{\mathrm{sg}}(R)$ the singularity category of $R$. This category has been introduced and studied by Buchweitz [1986] in connection with Cohen-Macaulay modules over Gorenstein rings. In recent years, it has been investigated by Orlov $[2004 ; 2006 ; 2009 a ; 2011 ; 2012]$ in relation to the homological mirror symmetry conjecture.

Let $\mathrm{S}(R)$ be the set of prime ideals $\mathfrak{p}$ of $R$ such that $R_{\mathfrak{p}}$ is not a field, and denote by Sing $R$ the singular locus of $R$. Applying Theorem 1.1, we can prove the following result on classification of subcategories.

Corollary 1.2. Let $R$ be a commutative noetherian ring.

(1) There is a one-to-one correspondence between:

(a) the specialization-closed subsets of $\mathrm{S}(R)$,

(b) the resolving subcategories of $\bmod R$ generated by a Serre subcategory of $\bmod R$.

(2) There are one-to-one correspondences among:

(a) the specialization-closed subsets of $\operatorname{Sing} R$,

(b) the thick subcategories of $\mathbf{D}_{\mathrm{b}}(R)$ generated by $R$ and a Serre subcategory of $\bmod R$,

(c) the thick subcategories of $\mathbf{D}_{\mathrm{sg}}(R)$ generated by a Serre subcategory of $\bmod R$. 
When $R$ is local, let $\bmod ^{\circ}(R)$ (respectively, $\mathbf{D}_{\mathrm{b}}^{\circ}(R), \mathbf{D}_{\mathrm{sg}}^{\circ}(R)$ ) be the full subcategories of $\bmod R$ (respectively, $\mathbf{D}_{\mathrm{b}}(R), \mathbf{D}_{\mathrm{sg}}(R)$ ) consisting of modules (respectively, complexes) that are locally free (respectively, perfect, zero) on the punctured spectrum of $R$. Applying Theorem 1.1, we can prove the following result on generation of subcategories.

Corollary 1.3. Let $R$ be a commutative noetherian local ring of Krull dimension d with residue field $k$.

(1) Every object in $\bmod ^{\circ}(R)$ is built out of a module of finite length by taking $d$ extensions in $\bmod R$, up to finite direct sums, direct summands and syzygies.

(2) Every object in $\mathbf{D}_{\mathrm{sg}}^{\circ}(R)$ is built out of a module of finite length by taking $d$ extensions in $\mathbf{D}_{\mathrm{sg}}(R)$, up to finite direct sums, direct summands and shifts.

In particular, one has that $\bmod ^{\circ}(R)$ is generated by $k$ as a resolving subcategory of $\bmod R$, that $\mathbf{D}_{\mathrm{b}}^{\circ}(R)$ is generated by $R$ and $k$ as a thick subcategory of $\mathbf{D}_{\mathrm{b}}(R)$, and that $\mathbf{D}_{\mathrm{sg}}^{\circ}(R)$ is generated by $k$ as a thick subcategory of $\mathbf{D}_{\mathrm{sg}}(R)$.

Corollary 1.3 yields variants of results shown by Schoutens [2003] and Takahashi [2009; 2010]. It also recovers a result on isolated singularities given by KellerMurfet-Van den Bergh [2011]. Furthermore, utilizing it, one can show the following result.

Corollary 1.4. Let $R$ be a commutative noetherian ring. The following are equivalent for a resolving subcategory $\mathscr{X}$ of $\bmod R$ :

(1) $\mathscr{L}$ is generated by a Serre subcategory of $\bmod R$.

(2) $\mathscr{X}$ is closed under tensor products and transposes.

Hence there is a one-to-one correspondence between the specialization-closed subsets of $\mathrm{S}(R)$ and the resolving subcategories of $\bmod R$ closed under tensor products and transposes.

The last assertion of this corollary greatly improves the main result of [Takahashi 2013]. Indeed, it removes the superfluous assumptions that $R$ is local and that $R$ is Cohen-Macaulay.

The organization of this paper is as follows. In the next Section 2 we prepare some fundamental notions. In Section 3 we state and prove the most general result in this paper, which includes Theorem 1.1. In the final Section 4 we apply the results shown in the preceding section to find out the structure of certain subcategories, and give several results including Corollaries 1.2, 1.3 and 1.4.

\section{Basic definitions}

This section is devoted to stating the definitions and basic properties of notions which we will freely use in the later sections. We begin with our convention. 
Convention 2.1. Throughout the present paper, let $R$ be a commutative noetherian ring with identity. We assume that all $R$-modules are finitely generated, that all $R$-complexes are homologically bounded, and that all subcategories of categories are full.

In what follows, $\mathscr{T}$ and $\mathscr{A}$ denote a triangulated category and an abelian category with enough projective objects, respectively.

Definition 2.2. (1) For a subcategory $\mathscr{X}$ of an additive category $\mathscr{C}$, the additive closure add $\mathscr{C} \mathscr{X}$ of $\mathscr{X}$ is defined to be the smallest subcategory of $\mathscr{C}$ containing $\mathscr{X}$ and closed under finite direct sums and direct summands.

(2) A Serre subcategory of $\mathscr{A}$ is defined to be a subcategory of $\mathscr{A}$ closed under subobjects, quotients and extensions.

(3) A thick subcategory of $\mathscr{T}$ is by definition a triangulated subcategory of $\mathscr{T}$ closed under direct summands. The thick closure of a subcategory $\mathscr{L}$ of $\mathscr{T}$ is defined as the smallest thick subcategory of $\mathscr{T}$ containing $\mathscr{X}$, and denoted by thick $\mathscr{T} \mathscr{X}$ or simply by thick $\mathscr{X}$. When $\mathscr{X}$ consists of a single object $M$, we denote it by thick $\mathscr{T} M$ or thick $M$.

(4) We denote by proj $\mathscr{A}$ the subcategory of $\mathscr{A}$ consisting of projective objects.

(5) Let $P=\left(\cdots \stackrel{d_{3}}{\rightarrow} P_{2} \stackrel{d_{2}}{\rightarrow} P_{1} \stackrel{d_{1}}{\rightarrow} P_{0} \rightarrow 0\right)$ be a projective resolution of $M \in \mathscr{A}$. Then for each $n>0$ we define the $n$-th syzygy $\Omega^{n} M$ of $M$ (with respect to $P$ ) as the image of $d_{n}$. This is uniquely determined up to projective summands.

(6) We define a resolving subcategory of $\mathscr{A}$ as a subcategory of $\mathscr{A}$ containing proj $\mathscr{A}$ and closed under direct summands, extensions and syzygies. The resolving closure of a subcategory $\mathscr{X}$ of $\mathscr{A}$ is by definition the smallest resolving subcategory of $\mathscr{A}$ containing $\mathscr{X}$, and denoted by res $\mathscr{A} \mathscr{X}$ or simply by res $\mathscr{X}$. When $\mathscr{X}$ consists of a single object $M$, we denote it by $\operatorname{res}_{\mathscr{A}} M$ or res $M$.

(7) Let $X, Y$ be complexes of objects of $\mathscr{A}$.

(a) A homomorphism $f: X \rightarrow Y$ of complexes is called a quasiisomorphism if the induced map $\mathrm{H}_{i}(f): \mathrm{H}_{i}(X) \rightarrow \mathrm{H}_{i}(Y)$ on the $i$-th homologies is an isomorphism for all integers $i$.

(b) We say that $X$ is equivalent to $Y$ if there exists a sequence $X^{0}, X^{1}, \ldots, X^{n}$ of complexes such that $X^{0}=X, X^{n}=Y$, and there is a quasiisomorphism between $X^{i}$ and $X^{i+1}$ for all $0 \leq i \leq n-1$. Then we write $X \simeq Y$.

Remark 2.3. (1) A Serre subcategory is defined for an arbitrary abelian category.

(2) A resolving subcategory is usually defined as a subcategory containing the projective objects and closed under direct summands, extensions and kernels of epimorphisms. This definition and ours are equivalent. 
(3) Let $\mathscr{X}$ be a resolving subcategory of $\mathscr{A}$. Let $M$ be an object of $\mathscr{X}$ and $n>0$ an integer. The $n$-th syzygy of $M$ with respect to some projective resolution of $M$ is in $\mathscr{X}$ if and only if the $n$-th syzygy of $M$ with respect to every projective resolution of $M$ is in $\mathscr{X}$.

We recall the notions of balls in $\mathcal{T}$ and $\mathscr{A}$ introduced in [Bondal and Van den Bergh 2003; Dao and Takahashi 2011; Rouquier 2008].

Definition 2.4. (1a) For a subcategory $\mathscr{X}$ of $\mathscr{T}$ we denote by $\langle\mathscr{X}\rangle$ the smallest subcategory of $\mathscr{T}$ containing $\mathscr{X}$ that is closed under finite direct sums, direct summands and shifts; in symbols, $\langle\mathscr{X}\rangle=\operatorname{add} \mathscr{T}\{X[i] \mid i \in \mathbb{Z}, X \in \mathscr{X}\}$. When $\mathscr{X}$ consists of a single object $M$, we simply denote it by $\langle M\rangle$.

(1b) For subcategories $\mathscr{X}, \mathscr{Y}$ of $\mathscr{T}$ we denote by $\mathscr{X} * \mathscr{Y}$ the subcategory of $\mathscr{T}$ consisting of objects $M$ which fits into an exact triangle $X \rightarrow M \rightarrow Y \rightsquigarrow$ in $\mathscr{T}$ with $X \in \mathscr{X}$ and $Y \in \mathscr{Y}$. We set $\mathscr{L} \diamond \mathscr{Y}=\langle\langle\mathscr{X}\rangle *\langle\mathscr{Y}\rangle\rangle$.

(1c) Let $\mathscr{C}$ be a subcategory of $\mathscr{T}$. We define the ball of radius $r$ centered at $\mathscr{C}$ as

$$
\langle\mathscr{C}\rangle_{r}= \begin{cases}\langle\mathscr{C}\rangle & (r=1), \\ \langle\mathscr{C}\rangle_{r-1} \diamond \mathscr{C}=\left\langle\langle\mathscr{C}\rangle_{r-1} *\langle\mathscr{C}\rangle\right\rangle & (r \geq 2) .\end{cases}
$$

If $\mathscr{C}$ consists of a single object $M$, then we simply denote it by $\langle M\rangle_{r}$. We write $\langle\mathscr{C}\rangle_{r}^{\mathscr{T}}$ when we should specify that $\mathscr{T}$ is the ground category where the ball is defined.

(2a) For a subcategory $\mathscr{X}$ of $\mathscr{A}$ we denote by [ $\mathscr{X}$ ] the smallest subcategory of $\mathscr{A}$ containing proj $\mathscr{A}$ and $\mathscr{X}$ that is closed under finite direct sums, direct summands and syzygies, that is, $[\mathscr{C}]=\operatorname{add}_{\mathscr{A}}\left(\operatorname{proj} \mathscr{A} \cup\left\{\Omega^{i} X \mid i \geq 0, X \in \mathscr{X}\right\}\right)$. When $\mathscr{X}$ consists of a single object $M$, we simply denote it by $[M]$.

(2b) For subcategories $\mathscr{X}, \mathscr{Y}$ of $\mathscr{A}$ we denote by $\mathscr{L} \circ \mathscr{y}$ the subcategory of $\mathscr{A}$ consisting of objects $M$ which fits into an exact sequence $0 \rightarrow X \rightarrow M \rightarrow Y \rightarrow 0$ in $\mathscr{A}$ with $X \in \mathscr{X}$ and $Y \in \mathscr{Y}$. We set $\mathscr{X} \bullet \mathscr{Y}=[[\mathscr{X}] \circ[\mathscr{Y}]]$.

(2c) Let $\mathscr{C}$ be a subcategory of $\mathscr{A}$. We define the ball of radius $r$ centered at $\mathscr{C}$ as

$$
[\mathscr{C}]_{r}= \begin{cases}{[\mathscr{C}]} & (r=1), \\ {[\mathscr{C}]_{r-1} \bullet \mathscr{C}=\left[[\mathscr{C}]_{r-1} \circ[\mathfrak{C}]\right]} & (r \geq 2) .\end{cases}
$$

If $\mathscr{C}$ consists of a single object $M$, then we simply denote it by $[M]_{r}$. We write $[\mathscr{C}]_{r}^{A}$ when we should specify that $\mathscr{A}$ is the ground category where the ball is defined.

Remark 2.5 [Bondal and Van den Bergh 2003; Dao and Takahashi 2011; Rouquier 2008]. (1) Let $\mathscr{X}, \mathscr{Y}, \mathscr{L}, \mathscr{C}$ be subcategories of $\mathscr{T}$.

(a) An object $M \in \mathscr{T}$ belongs to $\mathscr{L} \diamond \mathscr{Y}$ if and only if there is an exact triangle $X \rightarrow Z \rightarrow Y \rightsquigarrow$ with $X \in\langle\mathscr{X}\rangle, Y \in\langle\mathscr{Y}\rangle$, and $M$ a direct summand of $Z$.

(b) One has $(\mathscr{X} \diamond \mathscr{Y}) \diamond \mathscr{L}=\mathscr{X} \diamond(\mathscr{Y} \diamond \mathscr{L})$ and $\langle\mathscr{C}\rangle_{a} \diamond\langle\mathscr{C}\rangle_{b}=\langle\mathscr{C}\rangle_{a+b}$ for all $a, b>0$. 
(2) Let $\mathscr{X}, \mathscr{Y}, \mathscr{L}, \mathscr{C}$ be subcategories of $\mathscr{A}$.

(a) An object $M \in \mathscr{A}$ belongs to $\mathscr{X} \bullet \mathscr{Y}$ if and only if there is an exact sequence $0 \rightarrow X \rightarrow Z \rightarrow Y \rightarrow 0$ with $X \in[\mathscr{X}]$ and $Y \in[\mathscr{Y}]$ such that $M$ is a direct summand of $Z$.

(b) One has $(\mathscr{X} \bullet Y) \cdot \mathscr{L}=\mathscr{X} \bullet(\mathscr{Y} \bullet \mathscr{L})$ and $[\mathscr{C}]_{a} \bullet[\mathscr{C}]_{b}=[\mathscr{C}]_{a+b}$ for all $a, b>0$.

Definition 2.6. An $R$-complex is called perfect if it is a bounded complex of projective $R$-modules. The singularity category $\mathbf{D}_{\mathrm{sg}}(R)$ of $R$ is defined as the Verdier quotient of $\mathbf{D}_{\mathrm{b}}(R)$ by the perfect complexes. For the definition of a Verdier quotient, we refer to [Neeman 2001, Remark 2.1.9]. Whenever we discuss the singularity category $\mathbf{D}_{\mathrm{sg}}(R)$, we identify each object or subcategory of $\bmod R$ with its image in $\mathbf{D}_{\mathrm{sg}}(R)$ by the composition of the canonical functors $\bmod R \rightarrow \mathbf{D}_{\mathrm{b}}(R) \rightarrow \mathbf{D}_{\mathrm{sg}}(R)$.

Remark 2.7 [Dao and Takahashi 2012b, Lemma 2.4]. (1) For all $X \in \mathbf{D}_{\mathrm{b}}(R)$ there exists an exact triangle $P \rightarrow X \rightarrow M[n] \rightsquigarrow$ in $\mathbf{D}_{\mathrm{b}}(R)$ such that $P$ is a perfect complex, $M$ is a module and $n$ is an integer. In particular, $X \cong M[n]$ in $\mathbf{D}_{\mathrm{sg}}(R)$.

(2) For every $M \in \bmod R$ and every $n \geq 0$ there is an isomorphism $M \cong \Omega^{n} M[n]$ in $\mathbf{D}_{\mathrm{sg}}(R)$. Hence, for a subcategory $\mathscr{C}$ of $\bmod R$ and an integer $k>0$, each module in $[\mathscr{C}]_{k}^{\bmod R}$ belongs to $\langle\mathscr{C}\rangle_{k}^{\mathbf{D}_{\text {sg }}(R)}$.

We introduce subcategories which will be investigated in Section 4.

Definition 2.8. Let $\Phi$ be a subset of Spec $R$. Set $\Phi^{\mathrm{c}}=\operatorname{Spec} R \backslash \Phi$. We denote by $\mathrm{e}^{\Phi}(R)$ (respectively, $\bmod ^{\Phi}(R)$ ) the subcategory of $\bmod R$ consisting of $R$-modules $M$ such that $M_{\mathfrak{p}}=0$ (respectively, $M_{\mathfrak{p}}$ is $R_{\mathfrak{p}}$-free) for all $\mathfrak{p} \in \Phi^{\mathrm{c}}$. Also, $\mathbf{D}_{\mathrm{b}}^{\Phi}(R)$ (respectively, $\mathbf{D}_{\mathrm{sg}}^{\Phi}(R)$ ) denotes the subcategory of $\mathbf{D}_{\mathrm{b}}(R)$ (respectively, $\mathbf{D}_{\mathrm{sg}}(R)$ ) consisting of $R$-complexes $X$ such that $X_{\mathfrak{p}}$ isomorphic to a perfect $R_{\mathfrak{p}}$-complex in $\mathbf{D}_{\mathrm{b}}\left(R_{\mathfrak{p}}\right)$ (respectively, $X_{\mathfrak{p}} \cong 0$ in $\mathbf{D}_{\mathrm{sg}}\left(R_{\mathfrak{p}}\right)$ ) for all $\mathfrak{p} \in \Phi^{\mathrm{c}}$. We have that $\mathrm{e}^{\Phi}(R)$ is a Serre subcategory of $\bmod R$, that $\bmod ^{\Phi}(R)$ is a resolving subcategory of $\bmod R$, and that $\mathbf{D}_{\mathrm{b}}^{\Phi}(R), \mathbf{D}_{\mathrm{sg}}^{\Phi}(R)$ are thick subcategories of $\mathbf{D}_{\mathrm{b}}(R), \mathbf{D}_{\mathrm{sg}}(R)$ respectively.

Definition 2.9. (1) For an $R$-module $M$ we denote by $N F(M)$ the nonfree locus of $M$, that is, the set of prime ideals $\mathfrak{p}$ of $R$ such that the $R_{\mathfrak{p}}$-module $M_{\mathfrak{p}}$ is nonfree. As is well-known, $\operatorname{NF}(M)$ is a closed subset of Spec $R$ in the Zariski topology.

(2) For an $R$-complex $M$ we denote by $\operatorname{IPD}(M)$ the infinite projective dimension locus of $M$, that is, the set of prime ideals $\mathfrak{p}$ of $R$ such that the $R_{\mathfrak{p}}$-complex $M_{\mathfrak{p}}$ has infinite projective dimension.

(3) For a subcategory $\mathscr{X}$ of $\bmod R$ we set $\operatorname{Supp} \mathscr{X}=\bigcup_{M \in \mathscr{X}} \operatorname{Supp} M$ and NF( $\left.\mathscr{X}\right)=$ $\bigcup_{M \in \mathscr{L}} \mathrm{NF}(M)$.

(4) For a subcategory $\mathscr{X}$ of $\mathbf{D}_{\mathrm{b}}(R)$ we set IPD $(\mathscr{X})=\bigcup_{M \in \mathscr{L}} \operatorname{IPD}(M)$.

(5) For a subcategory $\mathscr{X}$ of $\mathbf{D}_{\mathrm{sg}}(R)$ we set $\operatorname{Supp}_{\mathrm{sg}}(\mathscr{X})=\bigcup_{M \in \mathscr{X}} \operatorname{IPD}(M)$. 
Definition 2.10. (1) Let $M$ be an $R$-module.

(a) Let $\boldsymbol{x}$ be a sequence of elements of $R$. Then $\mathrm{K}(\boldsymbol{x}, M)$ denotes the Koszul complex of $M$ with respect to $\boldsymbol{x}$. We call $\mathrm{H}_{i}(\boldsymbol{x}, M):=\mathrm{H}_{i}(\mathrm{~K}(\boldsymbol{x}, M))$ the $i$-th Koszul homology $(i \in \mathbb{Z})$ and $\mathrm{H}(\boldsymbol{x}, M):=\bigoplus_{i \in \mathbb{Z}} \mathrm{H}_{i}(\boldsymbol{x}, M)$ the Koszul homology of $M$ with respect to $\boldsymbol{x}$.

(b) Let $P_{1} \stackrel{d}{\rightarrow} P_{0} \rightarrow M \rightarrow 0$ be a projective presentation of $M$. Then the cokernel of the $R$-dual map of $d$ is called the transpose of $M$ and denoted by $\operatorname{Tr} M$. This is uniquely determined up to projective summands.

(2) A subset $\Phi$ of Spec $R$ is called specialization-closed if $\mathrm{V}(\mathfrak{p}) \subseteq \Phi$ for all $\mathfrak{p} \in \Phi$. This is nothing but a union of closed subsets of Spec $R$ in the Zariski topology.

(3) We denote by $\operatorname{Sing} R$ the singular locus of $R$, namely, the set of prime ideals $\mathfrak{p}$ of $R$ such that $R_{\mathfrak{p}}$ is not a regular local ring.

(4) A local ring $R$ with maximal ideal $\mathfrak{m}$ is called an isolated singularity if Sing $R \subseteq\{\mathfrak{m}\}$.

\section{Reconstruction from Koszul homology}

In this section, we consider reconstructing a given module from its Koszul homology by taking direct summands, extensions and syzygies. We start by stating and proving the most general result in this paper; actually, almost all of the other results given in this paper are deduced from this.

Theorem 3.1. Let $M$ be an $R$-module. Let $\boldsymbol{x}=x_{1}, \ldots, x_{n}$ be a sequence of elements of $R$ such that $x_{p} \operatorname{Ext}_{R}^{q}\left(M, \Omega^{r} M\right)=0$ for all $1 \leq p \leq n$ and $1 \leq q, r \leq p$. Let $P$ be a projective resolution of $M$. Then $\mathrm{K}(\boldsymbol{x}, M)$ is equivalent to a complex

$$
X=\left(0 \rightarrow X_{n} \rightarrow X_{n-1} \rightarrow \cdots \rightarrow X_{1} \rightarrow X_{0} \rightarrow 0\right)
$$

such that $X_{i}=\bigoplus_{j=0}^{i} P_{j}{ }^{\oplus\left(\begin{array}{c}n \\ i-j\end{array}\right)}$ for each $0 \leq i \leq n-1$ and $X_{n}=\bigoplus_{j=0}^{n}\left(\Omega^{j} M\right)^{\oplus\left(\begin{array}{c}n \\ j\end{array}\right)}$.

Proof. We prove the theorem by induction on $n$. Let us first consider the case where $n=1$. Multiplication by $x_{1}$ makes a pullback diagram:

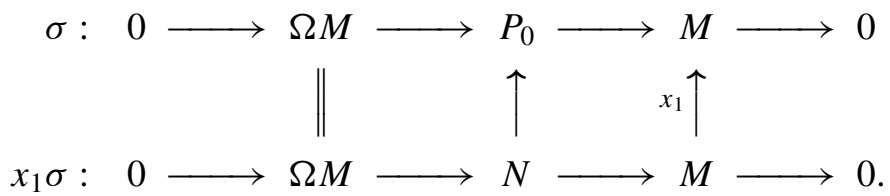

Since $x_{1} \operatorname{Ext}_{R}^{1}(M, \Omega M)=0$, we see that the exact sequence $x_{1} \sigma$ splits and get an isomorphism $N \cong \Omega M \oplus M$. Thus we obtain a short exact sequence of complexes

$$
0 \rightarrow W \rightarrow X \rightarrow \mathrm{K}\left(x_{1}, M\right) \rightarrow 0,
$$


where $W=(0 \rightarrow \Omega M \stackrel{=}{\rightarrow} \Omega M \rightarrow 0)$ and $X=\left(0 \rightarrow \Omega M \oplus M \rightarrow P_{0} \rightarrow 0\right)$. As $W$ is acyclic, $\mathrm{K}\left(x_{1}, M\right)$ is equivalent to $X$.

Next we assume $n \geq 2$. The induction hypothesis implies that $\mathrm{K}\left(x_{1}, \ldots, x_{n-1}, M\right)$ is equivalent to a complex

$$
Y=\left(0 \rightarrow Y_{n-1} \stackrel{f}{\rightarrow} Y_{n-2} \rightarrow \cdots \rightarrow Y_{1} \rightarrow Y_{0} \rightarrow 0\right)
$$

with $Y_{i}=\bigoplus_{j=0}^{i} P_{j}^{\oplus}\left(\begin{array}{c}n-1 \\ i-j\end{array}\right)$ for $0 \leq i \leq n-2$ and $Y_{n-1}=\bigoplus_{j=0}^{n-1}\left(\Omega^{j} M\right)^{\oplus\left(\begin{array}{c}n-1 \\ j\end{array}\right)}$. In general, taking a tensor product with a perfect complex preserves equivalence of complexes (cf. [Christensen 2000, A.4.1]). Hence we have

$$
\begin{aligned}
& \mathrm{K}(\boldsymbol{x}, M) \\
& \quad=\mathrm{K}\left(x_{1}, \ldots, x_{n-1}, M\right) \otimes_{R} \mathrm{~K}\left(x_{n}, R\right) \simeq Y \otimes_{R} \mathrm{~K}\left(x_{n}, R\right) \\
& \quad=\left(0 \rightarrow Y_{n-1} \stackrel{g}{\rightarrow} Y_{n-1} \oplus Y_{n-2} \stackrel{d_{n-1}}{\rightarrow} Y_{n-2} \oplus Y_{n-3} \stackrel{d_{n-2}}{\rightarrow} \cdots \stackrel{d_{2}}{\rightarrow} Y_{1} \oplus Y_{0} \stackrel{d_{1}}{\rightarrow} Y_{0} \rightarrow 0\right) \\
& \quad=: Z
\end{aligned}
$$

where $g=\left(\begin{array}{c}(-1)^{n-1} x_{n} \\ f\end{array}\right)$. Note that there is an exact sequence $0 \rightarrow \Omega Y_{n-1} \rightarrow Q \stackrel{\pi}{\rightarrow}$ $Y_{n-1} \rightarrow 0$ with $Q=\bigoplus_{j=0}^{n-1} P_{j} \oplus^{\oplus\left(\begin{array}{c}n-1 \\ j\end{array}\right)}$. Consider the pullback diagram

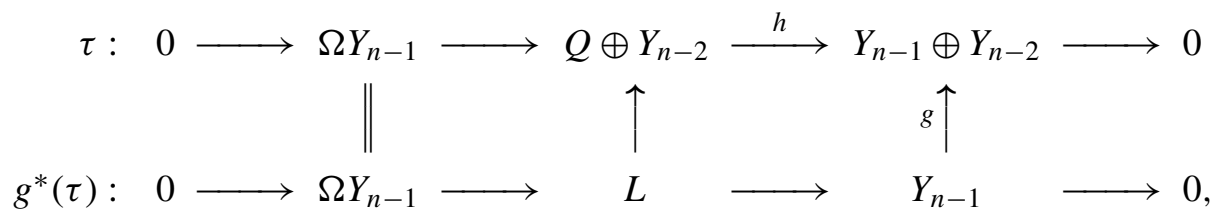

where $h=\left(\begin{array}{cc}\pi & 0 \\ 0 & 1\end{array}\right)$ and $g^{*}=\operatorname{Ext}_{R}^{1}\left(g, \Omega Y_{n-1}\right)$. As $Y_{n-2}$ is projective, the map $g^{*}$ can be identified with the multiplication map

$$
\operatorname{Ext}_{R}^{1}\left(Y_{n-1}, \Omega Y_{n-1}\right) \stackrel{(-1)^{n-1} x_{n}}{\longrightarrow} \operatorname{Ext}_{R}^{1}\left(Y_{n-1}, \Omega Y_{n-1}\right)
$$

There are isomorphisms

$$
\begin{aligned}
\operatorname{Ext}_{R}^{1}\left(Y_{n-1}, \Omega Y_{n-1}\right) & \cong \bigoplus_{j, k=0}^{n-1} \operatorname{Ext}_{R}^{1}\left(\Omega^{j} M, \Omega\left(\Omega^{k} M\right)\right)^{\oplus\left(\left(\begin{array}{c}
n-1 \\
j
\end{array}\right)+\left(\begin{array}{c}
n-1 \\
k
\end{array}\right)\right)} \\
& \cong \bigoplus_{j, k=0}^{n-1} \operatorname{Ext}_{R}^{j+1}\left(M, \Omega^{k+1} M\right)^{\oplus\left(\left(\begin{array}{c}
n-1 \\
j
\end{array}\right)+\left(\begin{array}{c}
n-1 \\
k
\end{array}\right)\right)}
\end{aligned}
$$

and hence $x_{n}$ annihilates $\operatorname{Ext}_{R}^{1}\left(Y_{n-1}, \Omega Y_{n-1}\right)$. Therefore $g^{*}(\tau)$ is a split exact sequence, and we obtain a commutative diagram

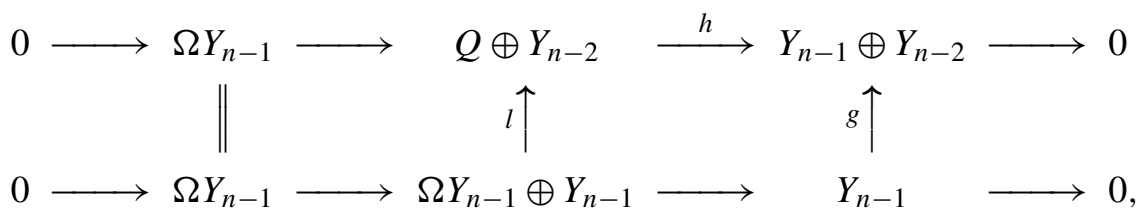


with exact rows. We observe that the complex $Z$ is equivalent to the complex

$$
\begin{aligned}
X= & \left(0 \rightarrow \Omega Y_{n-1} \oplus Y_{n-1} \stackrel{l}{\rightarrow} Q \oplus Y_{n-2} \stackrel{d_{n-1} h}{\rightarrow} Y_{n-2} \oplus Y_{n-3} \stackrel{d_{n-2}}{\longrightarrow}\right. \\
& \left.\cdots \stackrel{d_{2}}{\rightarrow} Y_{1} \oplus Y_{0} \stackrel{d_{1}}{\rightarrow} Y_{0} \rightarrow 0\right) .
\end{aligned}
$$

There are equalities

$$
\begin{aligned}
\Omega Y_{n-1} \oplus Y_{n-1} & =\bigoplus_{j=0}^{n}\left(\Omega^{j} M\right)^{\oplus\left(\begin{array}{c}
n \\
j
\end{array}\right),} \\
Q \oplus Y_{n-2} & =\bigoplus_{j=0}^{n-1} P_{j}^{\oplus\left(\begin{array}{c}
n \\
(n-1)-j
\end{array}\right),} \\
Y_{i} \oplus Y_{i-1} & =\bigoplus_{j=0}^{i} P_{j}^{\oplus\left(\begin{array}{c}
n \\
i-j
\end{array}\right)}
\end{aligned}
$$

for $1 \leq i \leq n-2$ and $Y_{0}=P_{0}$. Thus we are done.

Using Theorem 3.1, we obtain the following corollary.

Corollary 3.2. Let $M$ and $\boldsymbol{x}$ be as in Theorem 3.1.

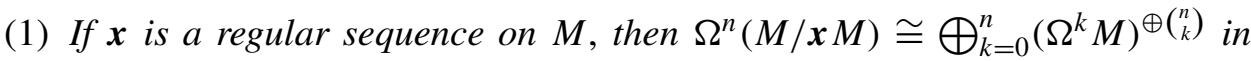
$\bmod R$.

(2) For each $1 \leq i \leq n$ there exists an exact sequence of $R$-modules

$$
0 \rightarrow \mathrm{H}_{i}(\boldsymbol{x}, M) \rightarrow E_{i} \rightarrow \Omega E_{i-1} \rightarrow 0
$$

with $E_{0}=\mathrm{H}_{0}(\boldsymbol{x}, M)$ such that $M$ is a direct summand of $E_{n}$. Hence $M$ is built out of $\mathrm{H}_{0}(\boldsymbol{x}, M), \ldots, \mathrm{H}_{n}(\boldsymbol{x}, M)$ by taking $n$ syzygies, $n$ extensions and 1 direct summand. In particular, $M$ belongs to the ball $[\mathrm{H}(\boldsymbol{x}, M)]_{n+1}^{\bmod R}$.

(3) There is an exact triangle

$$
F \rightarrow \mathrm{K}(\boldsymbol{x}, M) \rightarrow \bigoplus_{k=0}^{n}\left(\Omega^{k} M\right)^{\oplus\left(\begin{array}{l}
n \\
k
\end{array}\right)}[n] \rightsquigarrow
$$

in $\mathbf{D}_{\mathrm{b}}(R)$, where $F=\left(0 \rightarrow F_{n-1} \rightarrow \cdots \rightarrow F_{0} \rightarrow 0\right)$ is a perfect complex.

(4) The module $M$ belongs to the ball $\langle R \oplus \mathrm{K}(\boldsymbol{x}, M)\rangle_{n+1}^{\mathbf{D}_{\mathrm{b}}(R)}$.

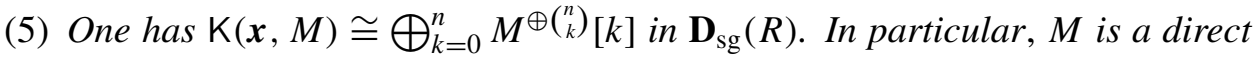
summand of $\mathrm{K}(\boldsymbol{x}, M)$ in $\mathbf{D}_{\mathrm{sg}}(R)$.

Proof. We use the notation of Theorem 3.1 and its assertion.

(1) Since $\boldsymbol{x}$ is regular on $M$, we have an equivalence $\mathrm{K}(\boldsymbol{x}, M) \simeq M / \boldsymbol{x} M$. There is an exact sequence

$$
0 \rightarrow X_{n} \rightarrow X_{n-1} \rightarrow \cdots \rightarrow X_{0} \rightarrow M / x M \rightarrow 0
$$

of $R$-modules. As $X_{n}=\bigoplus_{j=0}^{n}\left(\Omega^{j} M\right)^{\oplus\left(\begin{array}{c}n \\ j\end{array}\right)}$ and $X_{i}$ is projective for all $0 \leq i \leq n-1$, the module $X_{n}$ is the $n$-th syzygy of $M / x M$ as an $R$-module. 
(2) For each $0 \leq i \leq n$ take a truncation $X^{i}=\left(0 \rightarrow X_{n} \rightarrow \cdots \rightarrow X_{i+1} \rightarrow X_{i} \rightarrow 0\right)$ of $X$ with $\left(X^{i}\right)_{j}=X_{i+j}$ for $0 \leq j \leq n$. Then there is a short exact sequence

$$
0 \rightarrow X_{i-1} \rightarrow X^{i-1} \rightarrow X^{i}[1] \rightarrow 0
$$

of complexes for each $1 \leq i \leq n$. The long exact sequence in homology gives an exact sequence $0 \rightarrow \mathrm{H}_{1}\left(X^{i-1}\right) \rightarrow \mathrm{H}_{0}\left(X^{i}\right) \rightarrow X_{i-1} \rightarrow \mathrm{H}_{0}\left(X^{i-1}\right) \rightarrow 0$ of modules. As $X_{i-1}$ is projective, we have an exact sequence

$$
0 \rightarrow \mathrm{H}_{1}\left(X^{i-1}\right) \rightarrow \mathrm{H}_{0}\left(X^{i}\right) \rightarrow \Omega \mathrm{H}_{0}\left(X^{i-1}\right) \rightarrow 0
$$

for all $1 \leq i \leq n$. Notice $\mathrm{H}_{1}\left(X^{i-1}\right)=\mathrm{H}_{i}(\boldsymbol{x}, M), \mathrm{H}_{0}\left(X^{0}\right)=\mathrm{H}_{0}(\boldsymbol{x}, M)$ and $\mathrm{H}_{0}\left(X^{n}\right)=$ $X_{n}$. Setting $E_{i}=\mathrm{H}_{0}\left(X^{i}\right)$ for $0 \leq i \leq n$, we obtain desired exact sequences.

(3) Truncating the complex $X$ provides such an exact triangle.

(4) Decomposing $F$ into short exact sequences of complexes, we observe that $F$ is

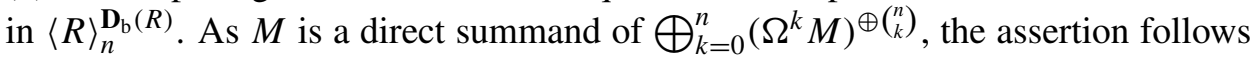
from (3).

(5) By (3) we have an isomorphism $\mathrm{K}(\boldsymbol{x}, M) \cong \bigoplus_{k=0}^{n}\left(\Omega^{k} M\right)^{\oplus\left(\begin{array}{l}n \\ k\end{array}\right)}[n]$ in $\mathbf{D}_{\mathrm{sg}}(R)$. Since $M \cong \Omega^{k} M[k]$ in $\mathbf{D}_{\text {sg }}(R)$, we are done.

Remark 3.3. (1) Corollary 3.2(1) is a refinement of [Takahashi 2010, Proposition 2.2], which shows the same conclusion under the additional assumption that $\boldsymbol{x}$ is a regular sequence on $R$ annihilating more Ext modules.

(2) Corollary 3.2(5) can also be shown by using the proof of [Dao and Takahashi 2012b, Proposition 2.3]. It also implies that $M$ belongs to $\langle R \oplus \mathrm{K}(\boldsymbol{x}, M)\rangle_{m}^{\mathbf{D}_{\mathrm{b}}(R)}$ for some integer $m>0$. However, it cannot determine how big/small $m$ is, while Corollary 3.2(4) can.

We are interested in existence of a sequence $\boldsymbol{x}$ as in Theorem 3.1. The lemma below guarantees that such a sequence always exists. Moreover, one can make such a sequence as a power of an arbitrary sequence whose defining closed subset covers the nonfree locus.

Lemma 3.4. Let $M$ be an $R$-module. Let $\boldsymbol{x}=x_{1}, \ldots, x_{n}$ be a sequence of elements of $R$ with $\mathrm{NF}(M) \subseteq \mathrm{V}(\boldsymbol{x})$. Then there exists an integer $k>0$ such that the sequence $\boldsymbol{x}^{k}=x_{1}^{k}, \ldots, x_{n}^{k}$ annihilates $\operatorname{Ext}_{R}^{i}(M, N)$ for all $i>0$ and all $N \in \bmod R$.

Proof. Let $I$ be an ideal of $R$ with $\operatorname{NF}(M)=\mathrm{V}(I)$. Then by [Dao and Takahashi 2012a, Remark 5.2(1)] there exists an integer $p>0$ such that $I^{p} \operatorname{Ext}_{R}^{i}(M, N)=0$ for all $i>0$ and all $N \in \bmod R$. By assumption, we have $\left(\boldsymbol{x}^{q}\right) \subseteq I$ for some $q>0$. Setting $k=p q$ completes the proof.

Combining Theorem 3.1, Corollary 3.2(2) and Lemma 3.4, we immediately obtain the following result, which includes Theorem 1.1. 
Corollary 3.5. Let $M$ be an $R$-module. Let $\boldsymbol{x}=x_{1}, \ldots, x_{n}$ be a sequence of elements of $R$ with $\mathrm{NF}(M) \subseteq \mathrm{V}(\boldsymbol{x})$. Then there exists an integer $k>0$ such that $\mathrm{K}\left(\boldsymbol{x}^{k}, M\right)$ is equivalent to a complex

$$
\left(0 \rightarrow N \rightarrow P_{n-1} \rightarrow \cdots \rightarrow P_{0} \rightarrow 0\right)
$$

where each $P_{i}$ is projective and $M$ is a direct summand of $N$. Hence, $M$ is built out of $\mathrm{H}_{0}\left(\boldsymbol{x}^{k}, M\right), \ldots, \mathrm{H}_{n}\left(\boldsymbol{x}^{k}, M\right)$ by taking $n$ syzygies, $n$ extensions and 1 direct summand. In particular, $M$ is in $\left[\mathrm{H}\left(\boldsymbol{x}^{k}, M\right)\right]_{n+1}^{\bmod R}$.

\section{Generation of subcategories}

In this section, we apply our results obtained in the previous section to investigate generation of subcategories. To be precise, for a subset $\Phi$ of Spec $R$ we analyze the structure of the subcategories $\bmod ^{\Phi}(R), \mathbf{D}_{\mathrm{b}}^{\Phi}(R)$ and $\mathbf{D}_{\mathrm{sg}}^{\Phi}(R)$. We also consider classification of these subcategories.

First of all, we want to make a generator of $\bmod ^{\Phi}(R)$ as a resolving subcategory of mod $R$ and generators of $\mathbf{D}_{\mathrm{b}}^{\Phi}(R), \mathbf{D}_{\mathrm{sg}}^{\Phi}(R)$ as thick subcategories of $\mathbf{D}_{\mathrm{b}}(R), \mathbf{D}_{\mathrm{sg}}(R)$. In fact, $\mathrm{e}^{\Phi}(R)$ gives generators of these three subcategories:

Theorem 4.1. Let $\Phi$ be a subset of $\operatorname{Spec} R$. Then one has equalities

$$
\begin{aligned}
\bmod ^{\Phi}(R) & =\operatorname{res}_{\bmod R}\left(\mathrm{e}^{\Phi}(R)\right), \\
\mathbf{D}_{\mathrm{b}}^{\Phi}(R) & =\operatorname{thick}_{\mathbf{D}_{\mathrm{b}}(R)}\left(\{R\} \cup \mathrm{e}^{\Phi}(R)\right), \\
\mathbf{D}_{\mathrm{sg}}^{\Phi}(R) & =\operatorname{thick}_{\mathbf{D}_{\mathrm{sg}}(R)}\left(\mathrm{e}^{\Phi}(R)\right) .
\end{aligned}
$$

Proof. (1) It is obvious that $\mathrm{e}^{\Phi}(R)$ is contained in $\bmod ^{\Phi}(R)$, and hence so is its resolving closure. To show the opposite inclusion, let $M$ be an object of $\bmod ^{\Phi}(R)$. Then by definition $\operatorname{NF}(M)$ is contained in $\Phi$. It is seen from Corollary 3.5 that there is a sequence $\boldsymbol{x}=x_{1}, \ldots, x_{n}$ of elements of $R$ with $\mathrm{NF}(M)=\mathrm{V}(\boldsymbol{x})$ such that $M$ belongs to res $\bmod _{\operatorname{mo}} \mathrm{H}(\boldsymbol{x}, M)$. Since $\mathrm{H}(\boldsymbol{x}, M)$ is annihilated by $\boldsymbol{x}$, we have

$$
\text { Supp } \mathrm{H}(\boldsymbol{x}, M) \subseteq \mathrm{V}(\boldsymbol{x})=\mathrm{NF}(M) \subseteq \Phi,
$$

which shows $\mathrm{H}(\boldsymbol{x}, M) \in \mathrm{e}^{\Phi}(R)$. Consequently, $M$ is in $\operatorname{res}_{\bmod R}\left(\mathrm{e}^{\Phi}(R)\right)$.

(2) Clearly, $\mathbf{D}_{\mathrm{b}}^{\Phi}(R)$ contains $R$ and $\mathrm{e}^{\Phi}(R)$, and the thick closure of $\{R\} \cup \mathrm{e}^{\Phi}(R)$. Let $X$ be an object of $\mathbf{D}_{\mathrm{b}}^{\Phi}(R)$. Then there is an exact triangle

$$
P \rightarrow X \rightarrow M[n] \rightsquigarrow
$$

in $\mathbf{D}_{\mathrm{b}}(R)$ such that $P$ is a perfect $R$-complex, $M$ is an $R$-module and $n$ is an integer. We use the large restricted flat dimension of $M$, namely

$$
\operatorname{Rfd}_{R} M=\sup _{\mathfrak{p} \in \operatorname{Spec} R}\left\{\text { depth } R_{\mathfrak{p}}-\operatorname{depth}_{R_{\mathfrak{p}}} M_{\mathfrak{p}}\right\}
$$


By [Avramov et al. 2010b, Theorem 1.1] this is finite. Put $r=\operatorname{Rfd}_{R} M$. Let $\mathfrak{p}$ be a prime ideal in $\Phi^{\mathrm{c}}$. Localizing the above exact triangle at $\mathfrak{p}$, we see that the $R_{\mathfrak{p}}$-module $M_{\mathfrak{p}}$ has finite projective dimension. Hence

$$
\operatorname{pd}_{R_{\mathfrak{p}}} M_{\mathfrak{p}}=\operatorname{depth} R_{\mathfrak{p}}-\operatorname{depth}_{R_{\mathfrak{p}}} M_{\mathfrak{p}} \leq r .
$$

Setting $N=\Omega^{r} M$, we note that $N$ belongs to $\bmod ^{\Phi}(R)$, hence to res $\bmod R\left(\mathrm{e}^{\Phi}(R)\right)$ by (1). Therefore $N$ is in thick $\mathbf{D}_{\mathrm{b}}(R)\left(\{R\} \cup \mathrm{e}^{\Phi}(R)\right)$, and so is $M$. As $P \in \operatorname{thick}_{\mathbf{D}_{\mathrm{b}}(R)} R$, the object $X$ belongs to thick $\mathbf{D}_{\mathrm{b}(R)}\left(\{R\} \cup \mathrm{e}^{\Phi}(R)\right)$ by the above exact triangle.

(3) This equality is obtained by using (2).

One can describe the structure of $\mathrm{e}^{\Phi}(R)$ in more detail, which makes more visible representations of $\bmod ^{\Phi}(R), \mathbf{D}_{\mathrm{b}}^{\Phi}(R)$ and $\mathbf{D}_{\mathrm{sg}}^{\Phi}(R)$.

Corollary 4.2. Let $\Phi$ be a subset of Spec $R$. Then $\mathrm{e}^{\Phi}(R)$ is the smallest subcategory of $\bmod R$ containing $R / \mathfrak{p}$ for all $\mathfrak{p} \in \Phi^{\mathrm{sp}}$ and closed under extensions. Here $\Phi^{\mathrm{sp}}$ denotes the largest specialization-closed subset of $\operatorname{Spec} R$ contained in $\Phi$. Hence

$$
\begin{aligned}
\bmod ^{\Phi}(R) & =\operatorname{res}_{\bmod R}\left\{R / \mathfrak{p} \mid \mathfrak{p} \in \Phi^{\mathrm{sp}}\right\}, \\
\mathbf{D}_{\mathrm{b}}^{\Phi}(R) & =\operatorname{thick}_{\mathbf{D}_{\mathrm{b}}(R)}\left\{R, R / \mathfrak{p} \mid \mathfrak{p} \in \Phi^{\mathrm{sp}}\right\}, \\
\mathbf{D}_{\mathrm{sg}}^{\Phi}(R) & =\operatorname{thick}_{\mathbf{D}_{\mathrm{sg}}(R)}\left\{R / \mathfrak{p} \mid \mathfrak{p} \in \Phi^{\mathrm{sp}}\right\} .
\end{aligned}
$$

Proof. The last assertion follows from Theorem 4.1.

We claim that $\Phi^{\mathrm{sp}}=\operatorname{Supp}\left(\mathrm{e}^{\Phi}(R)\right)$ holds. Indeed, it is evident that $\operatorname{Supp}\left(\mathrm{e}^{\Phi}(R)\right)$ is a specialization-closed subset of Spec $R$ contained in $\Phi$. Let $\Psi$ be a specializationclosed subset of Spec $R$ contained in $\Phi$. Then we have $\mathrm{e}^{\Psi}(R) \subseteq \mathrm{e}^{\Phi}(R)$, and hence $\Psi=\operatorname{Supp}\left(\mathrm{e}^{\Psi}(R)\right) \subseteq \operatorname{Supp}\left(\mathrm{e}^{\Phi}(R)\right)$. Thus the claim holds.

Let $\mathscr{X}$ be the smallest subcategory of $\bmod R$ containing $R / \mathfrak{p}$ for all $\mathfrak{p} \in \Phi^{\mathrm{sp}}$ and closed under extensions. First, let $\mathfrak{p}$ be a prime ideal in $\Phi^{\mathrm{sp}}$. As $\Phi^{\mathrm{sp}}$ is specializationclosed, we have $\operatorname{Supp}(R / \mathfrak{p})=\mathrm{V}(\mathfrak{p}) \subseteq \Phi^{\mathrm{sp}} \subseteq \Phi$, whence $R / \mathfrak{p}$ belongs to $\mathrm{e}^{\Phi}(R)$. Since $\mathrm{e}^{\Phi}(R)$ is closed under extensions, $\mathrm{e}^{\Phi}(R)$ contains $\mathscr{X}$. Next, let $M$ be a module in $\mathrm{e}^{\Phi}(R)$. Take a filtration

$$
M=M_{0} \supsetneq M_{1} \supsetneq \cdots \supsetneq M_{n}=0
$$

of submodules of $M$ such that $M_{i-1} / M_{i} \cong R / \mathfrak{p}_{i}$ with $\mathfrak{p}_{i} \in \operatorname{Spec} R$ for each $1 \leq i \leq n$. Then $\mathfrak{p}_{i}$ is in Supp $M$, and so in Supp $\left(\mathrm{e}^{\Phi}(R)\right)$. By the claim, we have $\mathfrak{p}_{i} \in \Phi^{\text {sp }}$ for all $1 \leq i \leq n$. Decomposing the above filtration into short exact sequences, we see that $M$ is in $\mathscr{X}$. Therefore $\mathscr{X}$ contains $\mathrm{e}^{\Phi}(R)$, and the proof is completed.

The next result, which includes part of Corollary 1.3, follows immediately from Corollary 4.2. Note that the objects of $\bmod ^{\{\mathfrak{m}\}}(R)$ are the $R$-modules that are locally free on the punctured spectrum of $R$. 
Corollary 4.3. (1) $\mathbf{D}_{\mathrm{b}}(R)=\operatorname{thick}\{R, R / \mathfrak{p} \mid \mathfrak{p} \in \operatorname{Sing} R\}$.

(2) $\mathbf{D}_{\mathrm{sg}}(R)=\operatorname{thick}\{R / \mathfrak{p} \mid \mathfrak{p} \in \operatorname{Sing} R\}$.

(3) If $R$ is a local ring with maximal ideal $\mathfrak{m}$ and residue field $k$, then Then $\bmod ^{\{\mathfrak{m}\}}(R)=\operatorname{res}(k), \mathbf{D}_{\mathrm{b}}^{\{\mathfrak{m}\}}(R)=\operatorname{thick}(R \oplus k)$ and $\mathbf{D}_{\mathrm{sg}}^{\{\mathfrak{m}\}}(R)=\operatorname{thick}(k)$.

Remark 4.4. The equalities in (1) and (2) can also be shown using Theorem VI.8 of [Schoutens 2003], while similar results to (3) have been obtained in Theorem 2.4 of [Takahashi 2010] as well as by H. Abe and O. Iyama (work in progress).

As a common consequence of the two assertions of Corollary 4.3, one can recover [Keller et al. 2011, Proposition A.2]:

Corollary 4.5. Let $R$ be an isolated singularity with residue field $k$. Then $\mathbf{D}_{\mathrm{b}}(R)=$ thick $(R \oplus k)$ and $\mathbf{D}_{\mathrm{sg}}(R)=\operatorname{thick}(k)$.

Next, we make a closer investigation on the inner structure of subcategories. In fact, we can refine the assertions as to $\bmod ^{\{\mathfrak{m}\}}(R)$ and $\mathbf{D}_{\mathrm{sg}}^{\{\mathfrak{m}\}}(R)$ in Corollary 4.3(3) in terms of balls in the abelian category $\bmod R$ and the triangulated category $\mathbf{D}_{\mathrm{sg}}(R)$. Denote by $\mathrm{fl}(R)$ the subcategory of $\bmod R$ consisting of modules of finite length. The following theorem holds, which is the main part of Corollary 1.3.

Theorem 4.6. Let $R$ be a d-dimensional local ring with maximal ideal $\mathfrak{m}$. Then there are equalities

$$
\bmod ^{\{\mathfrak{m}\}}(R)=[\mathrm{fl}(R)]_{d+1}^{\bmod R} \quad \text { and } \quad \mathbf{D}_{\mathrm{sg}}^{\{\mathfrak{m}\}}(R)=\langle\mathrm{fl}(R)\rangle_{d+1}^{\mathbf{D}_{\mathrm{sg}}(R)} .
$$

Proof. (1) Let us show the first equality. It clearly holds when $d=0$, so we assume $d>0$. Let $M$ be an $R$-module in $\bmod ^{\{\mathfrak{m}\}}(R)$. Take any system of parameters $\boldsymbol{x}=x_{1}, \ldots, x_{d}$ of $R$. As $M$ is in $\bmod ^{\{\mathfrak{m}\}}(R)$, we have $\mathrm{NF}(M) \subseteq\{\mathfrak{m}\}=\mathrm{V}(\boldsymbol{x})$. Corollary 3.5 implies that $M$ belongs to $\left[\mathrm{H}\left(x^{k}, M\right)\right]_{d+1}$ for some $k>0$. Since the $R$-module $\mathrm{H}\left(\boldsymbol{x}^{k}, M\right)$ is annihilated by the $\mathrm{m}$-primary ideal $\left(\boldsymbol{x}^{k}\right)$, it has finite length. Thus we obtain $M \in[\mathrm{fl}(R)]_{d+1}$, and the first equality follows.

(2) We prove the second equality. Let $X$ be an $R$-complex in $\mathbf{D}_{\mathrm{sg}}^{\{\mathfrak{m}\}}(R)$. Note that $X \cong \Omega^{d} M[n]$ in $\mathbf{D}_{\mathrm{sg}}(R)$ for some $R$-module $M$ and some integer $n$. By the Auslander-Buchsbaum formula, we see that $\Omega^{d} M$ belongs to $\bmod ^{\{\mathfrak{m}\}}(R)=[\mathrm{fl}(R)]_{d+1}$. Now the second equality follows from the first one.

Here is an immediate consequence of Theorem 4.6.

Corollary 4.7. If $R$ is a d-dimensional isolated singularity, $\mathbf{D}_{\mathrm{sg}}(R)=\langle\mathrm{fl}(R)\rangle_{d+1}$.

Remark 4.8. (1) Rewording the second equality in Theorem 4.6 by the terminology introduced in [Aihara et al. 2014], one has the following inequality:

$$
\mathrm{fl}(R)-\operatorname{tri} \cdot \operatorname{dim} \mathbf{D}_{\mathrm{sg}}^{\{\mathfrak{m}\}}(R) \leq \operatorname{dim} R .
$$


(2) Theorem A in [Takahashi 2009] constructs some object in $\bmod ^{\{\mathfrak{m}\}}(R)$ from every object in mod $R$ and counts the number of necessary operations (containing syzygies). In contrast to this, Theorem 4.6 constructs every object in $\bmod ^{\{\mathfrak{m}\}}(R)$ from some object in $\mathrm{fl}(R)$ and counts the number of necessary operations.

(3) Similar equalities to the first equality in Theorem 4.6 are given for $\bmod R$ in [Schoutens 2003, Theorem VI.8] and [Burke et al. 2012, Theorem 2], but these are different from ours in respect of how to count operations. The biggest difference is that neither of those two results counts the number of necessary extensions.

(4) In the case where $R$ is Cohen-Macaulay, Corollary 4.7 also follows from [Aihara et al. 2014, 4.5.1], because every maximal Cohen-Macaulay $R$-module is a direct summand of the $d$-th syzygy of some module of finite length by [Takahashi 2010, Proposition 2.2].

Finally, we are interested in classifying resolving and thick subcategories by using $\bmod ^{\Phi}(R), \mathbf{D}_{\mathrm{b}}^{\Phi}(R)$ and $\mathbf{D}_{\mathrm{sg}}^{\Phi}(R)$. For this purpose, we prepare a lemma:

Lemma 4.9. (1) The assignments $\mathscr{X} \mapsto$ Supp $\mathscr{X}$ and $\Phi \mapsto \mathrm{e}^{\Phi}(R)$ make a one-to-one correspondence between the Serre subcategories of $\bmod R$ and the specializationclosed subsets of Spec $R$.

(2) Let $\Phi$ be a specialization-closed subset of $\operatorname{Spec} R$. Then $\operatorname{NF}\left(\bmod ^{\Phi}(R)\right)=$ $\Phi \cap \mathrm{S}(R)$ and $\operatorname{IPD}\left(\mathbf{D}_{\mathrm{b}}^{\Phi}(R)\right)=\operatorname{Supp}_{\mathrm{sg}}\left(\mathbf{D}_{\mathrm{sg}}^{\Phi}(R)\right)=\Phi \cap \operatorname{Sing} R$.

Proof. (1) This is Gabriel's classification theorem [1962] for Serre subcategories.

(2) Let $\mathfrak{p} \in \Phi$. Then $\operatorname{IPD}(R / \mathfrak{p}) \subseteq \mathrm{NF}(R / \mathfrak{p}) \subseteq \mathrm{V}(\mathfrak{p}) \subseteq \Phi$. Hence $R / \mathfrak{p}$ belongs to $\bmod ^{\Phi}(R), \mathbf{D}_{\mathrm{b}}^{\Phi}(R)$ and $\mathbf{D}_{\mathrm{sg}}^{\Phi}(R)$. If $\mathfrak{p} \in \mathrm{S}(R)$ (respectively, Sing $R$ ), then $\mathfrak{p} \in \mathrm{NF}(R / \mathfrak{p})$ (respectively, $\operatorname{IPD}(R / \mathfrak{p}))$. The assertion now follows.

We can obtain the following theorem, which includes Corollary 1.2.

Theorem 4.10. (1) The assignment $\Phi \mapsto \bmod ^{\Phi}(R)$ is a bijection from the set of specialization-closed subsets of Spec $R$ contained in $\mathrm{S}(R)$ to the set of resolving closures res $\bmod R \mathscr{X}$, where $\mathscr{\mathscr { L }}$ runs through the Serre subcategories of $\bmod R$.

(2) The assignment $\Phi \mapsto \mathbf{D}_{\mathrm{b}}^{\Phi}(R)$ is a bijection from the set of specialization-closed subsets of $\operatorname{Spec} R$ contained in Sing $R$ to the set of thick closures thick $\mathbf{D}_{\mathbf{D}_{\mathrm{b}}(R)}(\{R\} \cup \mathscr{X})$, where $\mathscr{X}$ runs through the Serre subcategories of $\bmod R$.

(3) The assignment $\Phi \mapsto \mathbf{D}_{\mathrm{sg}}^{\Phi}(R)$ is a bijection from the set of specialization-closed subsets of Spec $R$ contained in $\operatorname{Sing} R$ to the set of thick closures thick $\mathbf{D}_{\mathbf{D}_{\mathrm{gg}}(R)} \mathscr{X}$, where $\mathscr{L}$ runs through the Serre subcategories of $\bmod R$.

Proof. In view of Theorem 4.1, the three assignments make well-defined maps, and they are injective by Lemma 4.9(2). Thus it only remains to show that they are surjective. 
(1) Let $\mathscr{L}$ be a Serre subcategory of $\bmod R$. According to Lemma 4.9(1), we have $\mathscr{X}=\mathrm{e}^{Z}(R)$ for some specialization-closed subset $Z$ of Spec $R$. Putting $\Phi=$ $Z \cap \mathrm{S}(R)$, we easily see that $\Phi$ is a specialization-closed subset of Spec $R$ which is contained in $\mathrm{S}(R)$ and satisfies $\bmod ^{Z}(R)=\bmod ^{\Phi}(R)$. Theorem 4.1 implies $\operatorname{res}_{\bmod } R \mathscr{X}=\bmod ^{\Phi}(R)$.

(2), (3) We use the proof of (1). Set $\Psi=Z \cap \operatorname{Sing} R$. Then $\Psi$ is a specializationclosed subset of $\operatorname{Spec} R$ contained in $\operatorname{Sing} R$ such that the equalities $\mathbf{D}_{\mathrm{b}}^{Z}(R)=$ $\mathbf{D}_{\mathrm{b}}^{\Psi}(R)$ and $\mathbf{D}_{\mathrm{sg}}^{Z}(R)=\mathbf{D}_{\mathrm{sg}}^{\Psi}(R)$ hold. Hence the surjectivity of the map follows from Theorem 4.1.

The next statement subsumes Corollary 1.4 and also some earlier results: namely, (1) and the equivalence of (b)-(d) in (2) are proved in [Takahashi 2013, Theorem 1.1 and Proposition 4.6] under the assumption that $R$ is a Cohen-Macaulay local ring. Our results show that this assumption is superfluous.

Corollary 4.11. (1) The assignments $\Phi \mapsto \bmod ^{\Phi}(R)$ and $\mathscr{L} \mapsto N F(\mathscr{X})$ gives mutually inverse bijections between

(a) the specialization-closed subsets of $\operatorname{Spec} R$ contained in $\mathrm{S}(R)$, and

(b) the resolving subcategories of $\bmod R$ closed under tensor products and transposes.

(2) Let $\mathscr{L}$ be a resolving subcategory of $\bmod R$. Then the following are equivalent:

(a) $\mathscr{X}$ is the resolving closure of a Serre subcategory of $\bmod R$.

(b) $\mathscr{X}$ is closed under tensor products and transposes.

(c) $R / \mathfrak{p}$ belongs to $\mathscr{X}$ for all $\mathfrak{p} \in \mathrm{NF}(\mathscr{C})$.

(d) For all $\mathfrak{p} \in \mathrm{NF}(\mathscr{X})$ there exists $M \in \mathscr{X}$ such that $\kappa(\mathfrak{p})$ is a direct summand of $M_{\mathfrak{p}}$.

Proof. Recall that we have proved in Corollary 4.3(3) that if $R$ is a local ring with maximal ideal $\mathfrak{m}$ and residue field $k$, then the equality $\bmod ^{\{\mathfrak{m}\}}(R)=\operatorname{res}_{\bmod R}(k)$ holds. Hence, in view of [Dao and Takahashi 2014, Lemma 3.2], we see that all the ten assertions in [Takahashi 2013, Lemma 2.5] hold without the assumption that $R$ is Cohen-Macaulay. Therefore, it is observed from [Dao and Takahashi 2014, Proposition 3.3] and the proof of [Takahashi 2013, Proposition 3.1] that one can remove from [Takahashi 2013, Proposition 3.1] the two assumptions that $R$ is local and that $R$ is Cohen-Macaulay. Thus, the proof of [Takahashi 2013, Theorem 3.3] actually proves that the statement [Takahashi 2013, Theorem 3.3] holds without the assumption that $R$ is a Cohen-Macaulay local ring. Since [Takahashi 2013, Lemma 4.5] (respectively, [Takahashi 2013, Lemma 4.4]) is still valid for an arbitrary commutative noetherian ring (respectively, local ring) $R$, so are [Takahashi 2013, Proposition 4.6 and Theorem 4.7]. Now our Theorem 4.10 completes the proof. 


\section{Acknowledgments}

The author is grateful to Osamu Iyama for giving him helpful suggestions and letting him know about their results. He thanks Takuma Aihara and Xiao-Wu Chen for useful comments, and the referee for reading the paper carefully.

\section{References}

[Aihara and Takahashi 2011] T. Aihara and R. Takahashi, "Generators and dimensions of derived categories”, preprint, 2011. arXiv 1106.0205

[Aihara et al. 2014] T. Aihara, T. Araya, O. Iyama, R. Takahashi, and M. Yoshiwaki, "Dimensions of triangulated categories with respect to subcategories", J. Algebra 399 (2014), 205-219. MR 3144584

[Avramov et al. 2010a] L. L. Avramov, R.-O. Buchweitz, S. B. Iyengar, and C. Miller, "Homology of perfect complexes", Adv. Math. 223:5 (2010), 1731-1781. MR 2011k:13014 Zbl 1186.13006

[Avramov et al. 2010b] L. L. Avramov, S. B. Iyengar, and J. Lipman, "Reflexivity and rigidity for complexes, I: Commutative rings", Algebra Number Theory 4:1 (2010), 47-86. MR 2011k:13016 Zbl 1194.13017

[Ballard et al. 2012] M. Ballard, D. Favero, and L. Katzarkov, "Orlov spectra: bounds and gaps", Invent. Math. 189:2 (2012), 359-430. MR 2947547 Zbl 1266.14013

[Balmer 2002] P. Balmer, "Presheaves of triangulated categories and reconstruction of schemes", Math. Ann. 324:3 (2002), 557-580. MR 2003j:18016 Zbl 1011.18007

[Balmer 2005] P. Balmer, "The spectrum of prime ideals in tensor triangulated categories", J. Reine Angew. Math. 588 (2005), 149-168. MR 2007b:18012 Zbl 1080.18007

[Benson et al. 2011] D. J. Benson, S. B. Iyengar, and H. Krause, "Stratifying modular representations of finite groups", Ann. of Math. (2) 174:3 (2011), 1643-1684. MR 2846489 Zbl 1261.20057

[Bergh et al. 2010] P. A. Bergh, S. B. Iyengar, H. Krause, and S. Oppermann, "Dimensions of triangulated categories via Koszul objects", Math. Z. 265:4 (2010), 849-864. MR 2011f:18016 Zbl 1263.18006

[Bondal and Van den Bergh 2003] A. Bondal and M. Van den Bergh, "Generators and representability of functors in commutative and noncommutative geometry", Mosc. Math. J. 3:1 (2003), 1-36. MR 2004h:18009 Zbl 1135.18302

[Buchweitz 1986] R.-O. Buchweitz, "Maximal Cohen-Macaulay modules and Tate-cohomology over Gorenstein rings”, preprint, University of Hannover, 1986, http://hdl.handle.net/1807/16682.

[Burke et al. 2012] J. Burke, L. W. Christensen, and R. Takahashi, "Building modules from the singular locus", preprint, 2012. To appear in Math. Scand. arXiv 1210.0055

[Christensen 1998] J. D. Christensen, "Ideals in triangulated categories: phantoms, ghosts and skeleta", Adv. Math. 136:2 (1998), 284-339. MR 99g:18007 Zbl 0928.55010

[Christensen 2000] L. W. Christensen, Gorenstein dimensions, Lecture Notes in Math. 1747, Springer, Berlin, 2000. MR 2002e:13032 Zbl 0965.13010

[Dao and Takahashi 2011] H. Dao and R. Takahashi, "The radius of a subcategory of modules", preprint, 2011. To appear in Algebra Number Theory. arXiv 1111.2902

[Dao and Takahashi 2012a] H. Dao and R. Takahashi, "The dimension of a subcategory of modules", preprint, 2012. arXiv 1203.1955

[Dao and Takahashi 2012b] H. Dao and R. Takahashi, "Upper bounds for dimensions of singularity categories”, preprint, 2012. arXiv 1203.1683 
[Dao and Takahashi 2014] H. Dao and R. Takahashi, "Classification of resolving subcategories and grade consistent functions”, Int. Math. Res. Not (2014). arXiv 1202.5605

[Friedlander and Pevtsova 2007] E. M. Friedlander and J. Pevtsova, "П-supports for modules for finite group schemes", Duke Math. J. 139:2 (2007), 317-368. MR 2008g:14081 Zbl 1128.20031

[Gabriel 1962] P. Gabriel, "Des catégories abéliennes", Bull. Soc. Math. France 90 (1962), 323-448. MR 38 \#1144 Zbl 0201.35602

[Hopkins and Smith 1998] M. J. Hopkins and J. H. Smith, "Nilpotence and stable homotopy theory, II”, Ann. of Math. (2) 148:1 (1998), 1-49. MR 99h:55009 Zbl 0927.55015

[Hovey 2001] M. Hovey, "Classifying subcategories of modules", Trans. Amer. Math. Soc. 353:8 (2001), 3181-3191. MR 2002i:13007 Zbl 0981.13006

[Keller et al. 2011] B. Keller, D. Murfet, and M. Van den Bergh, "On two examples by Iyama and Yoshino”, Compos. Math. 147:2 (2011), 591-612. MR 2012c:13029 Zbl 1264.13016

[Krause 2008] H. Krause, "Thick subcategories of modules over commutative Noetherian rings (with an appendix by Srikanth Iyengar)", Math. Ann. 340:4 (2008), 733-747. MR 2008m:13017 Zbl 1143.13012

[Krause and Kussin 2006] H. Krause and D. Kussin, "Rouquier's theorem on representation dimension", pp. 95-103 in Trends in representation theory of algebras and related topics (Querétaro, 2004), edited by J. A. de la Peña and R. Bautista, Contemp. Math. 406, Amer. Math. Soc., Providence, RI, 2006. MR 2008c:16010 Zbl 1107.16013

[Krause and Stevenson 2013] H. Krause and G. Stevenson, "A note on thick subcategories of stable derived categories”, Nagoya Math. J. 212 (2013), 87-96. arXiv 1111.2220

[Neeman 1992] A. Neeman, "The chromatic tower for $D(R)$ ", Topology 31:3 (1992), 519-532. MR 93h:18018 Zbl 0793.18008

[Neeman 2001] A. Neeman, Triangulated categories, Ann. of Math. Stud. 148, Princeton University Press, 2001. MR 2001k:18010 Zbl 0974.18008

[Oppermann 2009] S. Oppermann, "Lower bounds for Auslander's representation dimension", Duke Math. J. 148:2 (2009), 211-249. MR 2010i:16018 Zbl 1173.16007

[Orlov 2004] D. O. Orlov, “Триангулированные категории особенностей и D-браны в моделях Ландау-Гинзбурга", Tr. Mat. Inst. Steklova 246 (2004), 240-262. Translated as "Triangulated categories of singularities and D-branes in Landau-Ginzburg models", Proc. Steklov Inst. Math. 246:3 (2004), 227-248. MR 2006i:81173 Zbl 1101.81093 arXiv math/0302304

[Orlov 2006] D. O. Orlov, “Триангулированные категории особенностей и эквивалентности между моделями Ландау-Гинзбурга”, Mat. Sb. 197:12 (2006), 117-132. Translated as "Triangulated categories of singularities, and equivalences between Landau-Ginzburg models", Sb. Math. 197:12 (2006), 1827-1840. MR 2009g:14013 Zbl 1161.14301

[Orlov 2009a] D. O. Orlov, "Derived categories of coherent sheaves and triangulated categories of singularities", pp. 503-531 in Algebra, arithmetic, and geometry: in honor of Yu. I. Manin, vol. II, edited by Y. Tschinkel and Y. G. Zarhin, Progr. Math. 270, Birkhäuser, Boston, 2009. MR 2011c:14050 Zbl 1200.18007

[Orlov 2009b] D. O. Orlov, "Remarks on generators and dimensions of triangulated categories", Mosc. Math. J. 9:1 (2009), 143-149. MR 2011a:14031 Zbl 1197.18004

[Orlov 2011] D. O. Orlov, "Formal completions and idempotent completions of triangulated categories of singularities", Adv. Math. 226:1 (2011), 206-217. MR 2012c:14035 Zbl 1216.18012

[Orlov 2012] D. O. Orlov, "Matrix factorizations for nonaffine LG-models", Math. Ann. 353:1 (2012), 95-108. MR 2910782 Zbl 1243.81178 
[Rouquier 2006] R. Rouquier, "Representation dimension of exterior algebras", Invent. Math. 165:2 (2006), 357-367. MR 2007f:16031 Zbl 1101.18006

[Rouquier 2008] R. Rouquier, "Dimensions of triangulated categories", J. K-Theory 1:2 (2008), 193-256. MR 2009i:18008 Zbl 1165.18008

[Schoutens 2003] H. Schoutens, "Projective dimension and the singular locus", Comm. Algebra 31:1 (2003), 217-239. MR 2005e:13020 Zbl 1014.13003

[Stevenson 2014] G. Stevenson, "Subcategories of singularity categories via tensor actions", Compos. Math (2014). arXiv 1105.4698

[Takahashi 2009] R. Takahashi, "Modules in resolving subcategories which are free on the punctured spectrum”, Pacific J. Math. 241:2 (2009), 347-367. MR 2010b:13027 Zbl 1172.13005

[Takahashi 2010] R. Takahashi, "Classifying thick subcategories of the stable category of CohenMacaulay modules”, Adv. Math. 225:4 (2010), 2076-2116. MR 2011h:13014 Zbl 1202.13009

[Takahashi 2013] R. Takahashi, "Classifying resolving subcategories over a Cohen-Macaulay local ring”, Math. Z. 273:1-2 (2013), 569-587. MR 3010176 Zbl 1267.13024

[Thomason 1997] R. W. Thomason, "The classification of triangulated subcategories", Compos. Math. 105:1 (1997), 1-27. MR 98b:18017 Zbl 0873.18003

Received June 12, 2013.

RYO TAKAHASHI

GRaduate School of Mathematics

NAGOYA UNIVERSITY

FUROCHO, CHIKUSAKU

NAGOYA 464-8602

JAPAN

takahashi@math.nagoya-u.ac.jp 


\title{
PACIFIC JOURNAL OF MATHEMATICS
}

\author{
msp.org/pjm
}

Founded in 1951 by E. F. Beckenbach (1906-1982) and F. Wolf (1904-1989)

\section{EDITORS}

V. S. Varadarajan (Managing Editor)

Department of Mathematics

University of California

Los Angeles, CA 90095-1555

pacific@math.ucla.edu

Paul Balmer

Department of Mathematics

University of California

Los Angeles, CA 90095-1555

balmer@math.ucla.edu

Daryl Cooper

Department of Mathematics

University of California

Santa Barbara, CA 93106-3080 cooper@math.ucsb.edu

Jiang-Hua $\mathrm{Lu}$

Department of Mathematics

Pokfulam Rd., Hong Kong jhlu@maths.hku.hk
The University of Hong Kong

Don Blasius

Department of Mathematics University of California

Los Angeles, CA 90095-1555

blasius@math.ucla.edu

Robert Finn

Department of Mathematics Stanford University

Stanford, CA 94305-2125

finn@math.stanford.edu

Sorin Popa

Department of Mathematics

University of California

Los Angeles, CA 90095-1555 popa@math.ucla.edu

Paul Yang

Department of Mathematics Princeton University

Princeton NJ 08544-1000

yang@math.princeton.edu

\section{PRODUCTION}

Silvio Levy, Scientific Editor, production@msp.org

\section{SUPPORTING INSTITUTIONS}

ACADEMIA SINICA, TAIPEI

CALIFORNIA INST. OF TECHNOLOGY

INST. DE MATEMÁTICA PURA E APLICADA

KEIO UNIVERSITY

MATH. SCIENCES RESEARCH INSTITUTE

NEW MEXICO STATE UNIV.

OREGON STATE UNIV.

\author{
STANFORD UNIVERSITY \\ UNIV. OF BRITISH COLUMBIA \\ UNIV. OF CALIFORNIA, BERKELEY \\ UNIV. OF CALIFORNIA, DAVIS \\ UNIV. OF CALIFORNIA, LOS ANGELES \\ UNIV. OF CALIFORNIA, RIVERSIDE \\ UNIV. OF CALIFORNIA, SAN DIEGO \\ UNIV. OF CALIF., SANTA BARBARA
}

\author{
Vyjayanthi Chari \\ Department of Mathematics \\ University of California \\ Riverside, CA 92521-0135 \\ chari@math.ucr.edu \\ Kefeng Liu \\ Department of Mathematics \\ University of California \\ Los Angeles, CA 90095-1555 \\ liu@math.ucla.edu \\ Jie Qing \\ Department of Mathematics \\ University of California \\ Santa Cruz, CA 95064 \\ qing@cats.ucsc.edu
}

These supporting institutions contribute to the cost of publication of this Journal, but they are not owners or publishers and have no responsibility for its contents or policies.

See inside back cover or msp.org/pjm for submission instructions.

The subscription price for 2014 is US \$410/year for the electronic version, and \$535/year for print and electronic.

Subscriptions, requests for back issues and changes of subscribers address should be sent to Pacific Journal of Mathematics, P.O. Box 4163, Berkeley, CA 94704-0163, U.S.A. The Pacific Journal of Mathematics is indexed by Mathematical Reviews, Zentralblatt MATH, PASCAL CNRS Index, Referativnyi Zhurnal, Current Mathematical Publications and Web of Knowledge (Science Citation Index).

The Pacific Journal of Mathematics (ISSN 0030-8730) at the University of California, c/o Department of Mathematics, 798 Evans Hall \#3840, Berkeley, CA 94720-3840, is published twelve times a year. Periodical rate postage paid at Berkeley, CA 94704, and additional mailing offices. POSTMASTER: send address changes to Pacific Journal of Mathematics, P.O. Box 4163, Berkeley, CA 94704-0163.

PJM peer review and production are managed by EditFLOW ${ }^{\circledR}$ from Mathematical Sciences Publishers.

\section{PUBLISHED BY}

mathematical sciences publishers

nonprofit scientific publishing

http://msp.org/

(C) 2014 Mathematical Sciences Publishers 


\section{PACIFIC JOURNAL OF MATHEMATICS}

Volume $268 \quad$ No. $1 \quad$ March 2014

AlEXANDRE PAIVA BARRETO

A transport inequality on the sphere obtained by mass transport

DARIO CORDERO-ERAUSQUIN

A cohomological injectivity result for the residual automorphic spectrum of $\mathrm{GL}_{n}$

HARALD GROBNER

Gradient estimates and entropy formulae of porous medium and fast diffusion equations for the Witten Laplacian

GUANGYUE HUANG and HAIZHONG LI

Controlled connectivity for semidirect products acting on locally finite trees

KeITH JoNES

An indispensable classification of monomial curves in $\mathbb{A}^{4}(\mathbb{k})$

ANARGYROS KATSABEKIS and IGNACIO OJEDA

Contracting an axially symmetric torus by its harmonic mean curvature

CHRISTOPHER KIM

Composition operators on strictly pseudoconvex domains with smooth symbol

HYUNGWOON KOO and SONG-YING LI

The Alexandrov problem in a quotient space of $\mathbb{U}^{2} \times \mathbb{R}$

AnA Menezes

Twisted quantum Drinfeld Hecke algebras

DEEPAK NAIDU

$L^{p}$ harmonic 1-forms and first eigenvalue of a stable minimal hypersurface

KEOMKYO SEO

Reconstruction from Koszul homology and applications to module and derived categories

RYO TAKAHASHI

A virtual Kawasaki-Riemann-Roch formula 